\title{
Editorial
}

\section{Computational Intelligence Approaches to Robotics, Automation, and Control}

\author{
Yi Chen, ${ }^{1}$ Yun Li, ${ }^{2}$ Huosheng $\mathrm{Hu}^{3}{ }^{3}$ Jun Zhang, ${ }^{4}$ Dongbing $\mathrm{Gu},{ }^{3}$ and Peter $\mathrm{Xu}^{5}$ \\ ${ }^{1}$ School of Engineering and Built Environment, Glasgow Caledonian University, Glasgow G4 OBA, UK \\ ${ }^{2}$ School of Engineering, University of Glasgow, Glasgow G12 8QQ, UK \\ ${ }^{3}$ School of Computer Science and Electronic Engineering, University of Essex, Wivenhoe Park, Colchester CO4 3SQ, UK \\ ${ }^{4}$ Department of Computer Science, Sun Yat-Sen University, Guangzhou 510060, China \\ ${ }^{5}$ Department of Mechanical Engineering, University of Auckland, Private Bag 92019, Auckland, New Zealand
}

Correspondence should be addressed to Yi Chen; leo.chen.yi@live.co.uk

Received 11 September 2014; Accepted 11 September 2014

Copyright (C) 2015 Yi Chen et al. This is an open access article distributed under the Creative Commons Attribution License, which permits unrestricted use, distribution, and reproduction in any medium, provided the original work is properly cited.

Computational intelligence (CI) approaches are natureinspired methods, which offer a wealth of ideas for complex problems solving. Compared with the traditional approaches, the CI approaches are more powerful so that they do not need the reformulation of the problem to search a nonlinear and nondifferentiable space with real world conditions and need for massive parallelism. Another advantage of the $\mathrm{CI}$ is the flexibility of the fitness function formulation, which can be expressed as a proper function of the system's output and is suitable for multiobjective (MO) problems.

Robotics is a wide range research which includes design, construction, operation, and applications of robotic systems, as well as computer systems for control, sensory feedback, and information processing, in which CI approaches have been widely employed in automation and control that can take the place of humans in dangerous environments or manufacturing processes or resemble humans in appearance, behaviours, and cognition.

The special issue focuses on the theoretical, numeri$\mathrm{cal}$, and experimental contributions that describe original research results, innovative concepts that address all aspects of robotics, and CI approaches and are applying their results in the context of robotics, automation, and control. The aim is to establish a common understanding about the state of the field and draw a road map on where the research is heading, highlight the issues, and discuss the possible solutions.
Twenty-three papers are accepted to this special issue after a thorough reviewing process; the acceptance rate is $38.33 \%$ of all the submissions.

The special issue reports the continuing efforts to understand the research and development of CI approaches for optimisations and field applications, academic excellence, and ground-breaking research results in robotics, automation, and control systems, which is dedicated to researchers who wish to enhance or broaden their knowledge and expertise on academic and industrial leadership in robotics, high potential growth global robotics market, for example, industrial robotics and professional/domestic service robotics in the European Union, United States, China, and Japan.

\section{Acknowledgments}

The authors would like to express their faithful gratitude to all the contributors of this special issue for their support and to all the reviewers for their constructive and timely comments.

Yi Chen

Yun $\mathrm{Li}$

Huosheng $\mathrm{Hu}$ Jun Zhang

Dongbing $\mathrm{Gu}$

Peter Xu 


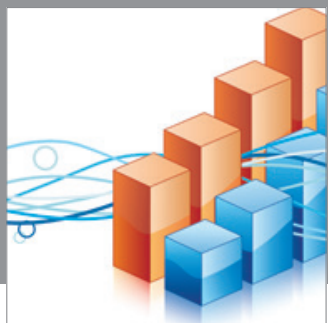

Advances in

Operations Research

mansans

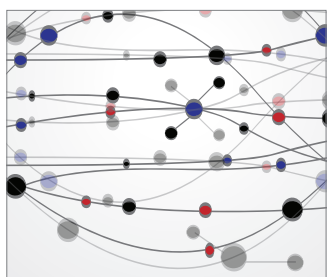

The Scientific World Journal
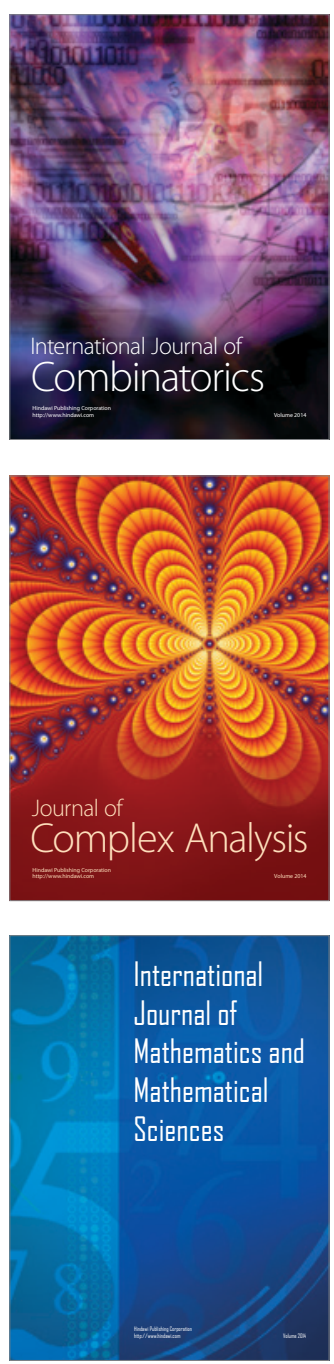
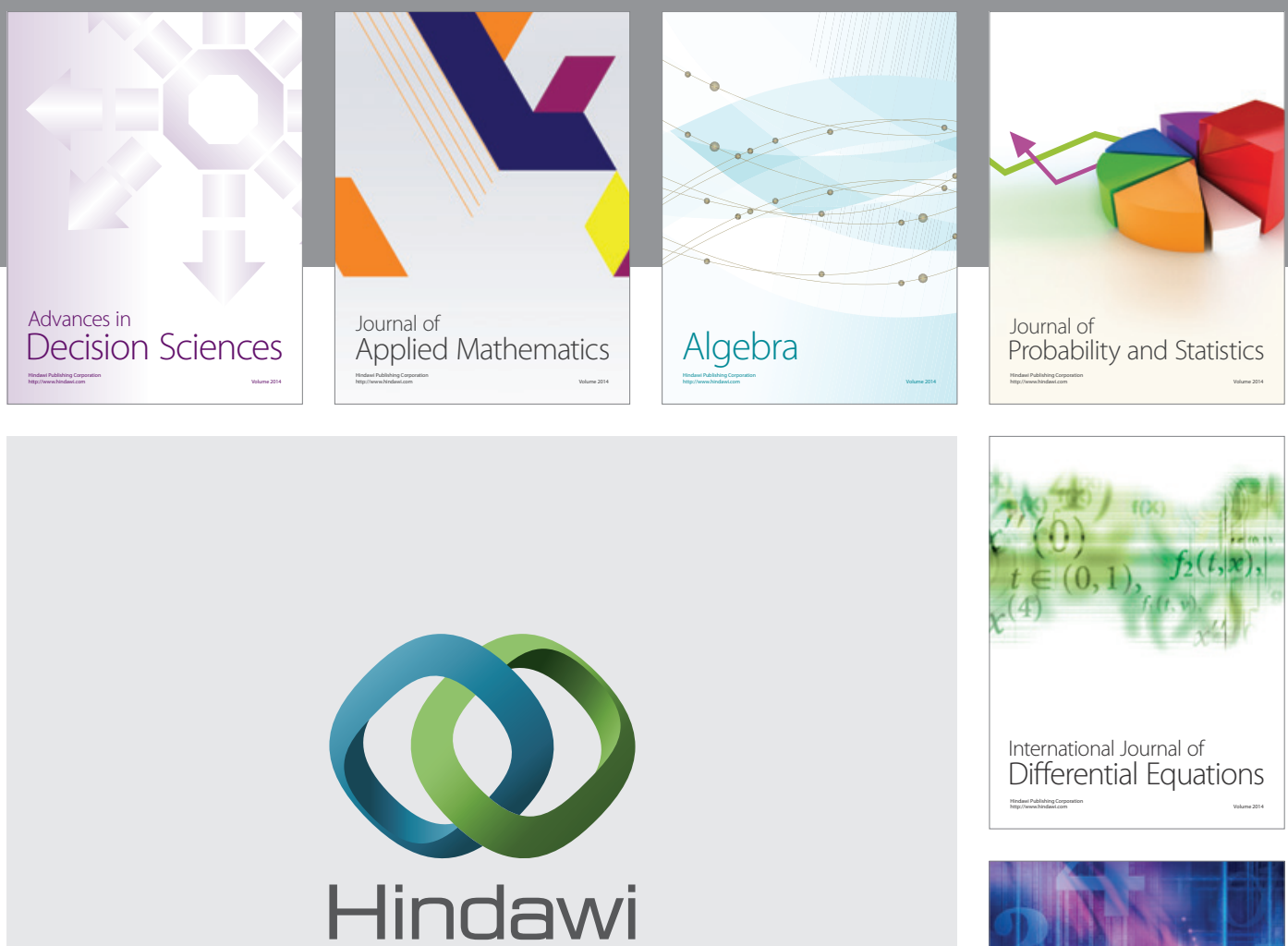

Submit your manuscripts at http://www.hindawi.com
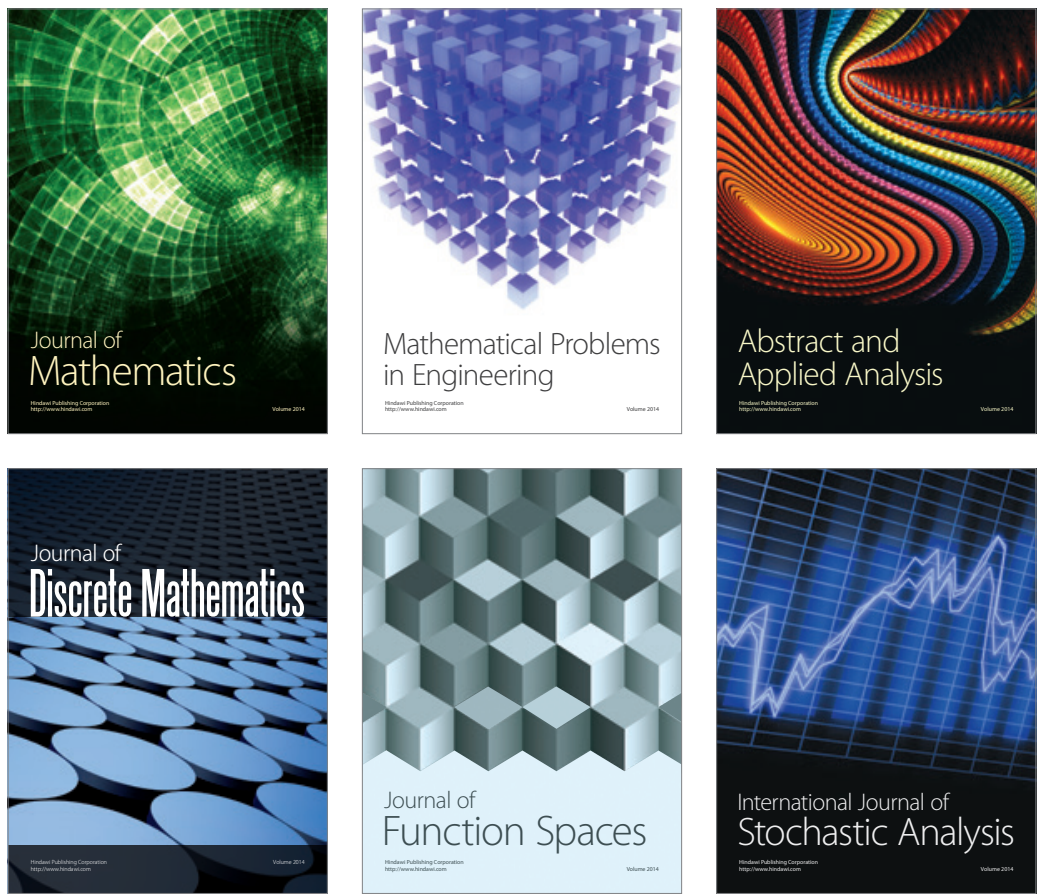

Journal of

Function Spaces

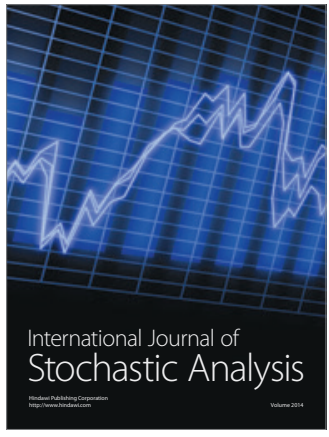

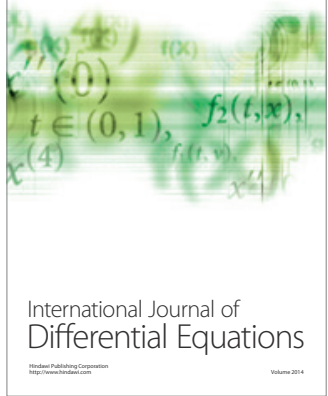
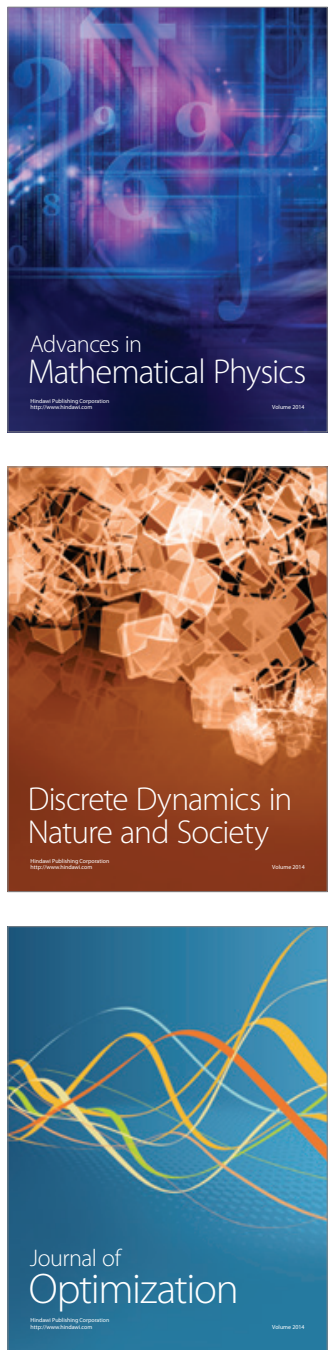